\title{
SUL PRIMO TEOREMA FONDAMENTALE DI LIE NELLA TEORIA DEI GRUPPI DI TRASFORMAZIONI.
}

\author{
Nota di Carlo Severini (Catania).
}

Adunanza del 12 maggio 1907.

Affinchè un insieme $\infty^{r}$ di trasformazioni

$$
\left.x_{i}^{\prime}=f_{i}(x, a)^{r}\right)
$$$$
(i=1,2, \ldots, n)
$$

costituisca un gruppo è sufficiente, come insegna il primo teorema fondamentale di LiE ${ }^{2}$ ) che le $x^{\prime}$, quali funzioni dei parametri $a$, soddisfino ad equazioni della forma:

$$
\frac{\partial x_{b}^{\prime}}{\partial a_{k}}=\sum_{p=1}^{p=r} \xi_{\rho h}\left(x^{\prime}\right) \psi_{p k}(a) \quad\left(\begin{array}{l}
b=1,2, \ldots, n \\
k=1,2, \ldots, r
\end{array}\right),
$$

e che fra le (I) sia contenuta la trasformazione identica, corrispondente a valori dei parametri, pei quali il determinante delle $\psi_{\rho k}(a)$ è diverso da zero.

La proposizione inversa non è vera in tutta la sua estensione, perchè, ammesso che le (I) costituiscano un gruppo, se si puó dire che esse soddisfano ad equazioni come le (2), in cui il determinante delle $\psi_{\rho k}(a)$ non è identicamente nullo, e le $\xi_{\rho h}\left(x^{\prime}\right)$ non soddisfano a nessun sistema di equazioni della forma:

$$
\sum_{\rho=1}^{\rho=r} g_{\rho} \xi_{\rho h}\left(x^{\prime}\right)=0
$$$$
(b=1,2, \ldots, n)
$$

coi coefficienti $g_{\rho}$ indipendenti dalle $x^{\prime}$ e non tutti nulli ${ }^{3}$ ), non puó peraltro asserirsi che all'insieme ( $I$ ) appartenga la trasformazione identica.

Se peró, essendo il campo di variabilità delle $x$ e delle $a$ il campo totale di tutti i possibili valori di siffatte variabili ${ }^{4}$ ), il determinante delle $\psi_{\rho k}(a)$, nelle equazioni (2), a cui supponiamo soddisfino le (I), risulta sempre diverso da zero, la trasforma-

1) Scriviamo per brevità, secondo l'uso, $f_{i}(x, a)$ invece di $f_{i}\left(x_{\mathrm{I}}, x_{2}, \ldots, x_{n} ; a_{\mathrm{I}}, a_{2}, \ldots, a_{\mathrm{r}}\right.$ ), ed analoghe notazioni useremo in casi analoghi.

2) Cfr. LIE, Theorie der Transformationsgruppen, III ${ }^{\text {ten }}$ Abschnit, Abth. VI (Leipzig, Teubner, I893).

3) Questa proprietà delle $\xi_{p h}\left(x^{\prime}\right)$ è nella prima parte del teorema di LIE implicita nella condizione che $\mathrm{i}$ parametri siano essenziali.

4) Per tale ipotesi le funzioni che avremo da considerare, le quali d'ordinario si suppongono analitiche regolari, s'intenderà che siano trascendenti intere. 
zione identica deve necessariamente essere contenuta fra queste, aflinchè possano costituire un gruppo.

Di ciò mi occupo anzitutto nella presente Nota. Indico poi, per il caso ora detto, delle condizioni che equivalgono all'ipotesi che esista la trasformazione identica, e che possono pertanto condurre a dimostrare che formano un gruppo le trasformazioni di un dato insieme, del quale si sa soltanto che soddisfano ad equazioni del tipo delle (2), insieme cui si puó pervenire colla diretta integrazione delle stesse (2), ove si fissi, mediante una trasformazione che non sia l'identica, il modo di dipendere delle $x^{\prime}$ dalle $x$, per un determinato sistema di valori dei parametri.

I. Ricordiamo che dall'essere i parametri essenziali si deduce che le funzioni $\xi_{p h}\left(x^{\prime}\right)$ non possono soddisfare ad equazioni come le (3), e che il determinante delle $\psi_{\rho k}(a)$ è in generale diverso da zero: noi ammetteremo, come dianzi abbiamo accennato, che sia sempre diverso da zero.

Se si pone:

$$
X_{\rho}^{\prime} f=\sum_{h=1}^{h=n} \xi_{\rho h}\left(x^{\prime}\right) \frac{\partial f}{\partial x_{b}^{\prime}}
$$$$
(p=1,2, \ldots, r)
$$

le $r$ trasformazioni infinitesimali $X_{\rho}^{\prime} f$ saranno linearmente indipendenti, e si avrà inoltre:

$$
\left(X_{k}^{\prime} X_{\rho}^{\prime}\right) f=\sum_{s=1}^{s=r} C_{k \rho s} X_{s}^{\prime} f \quad(k, p=\mathrm{I}, 2, \ldots, r ; \rho \neq k),
$$

ove i coefficienti $C_{k \rho s}$ sono quantità costanti.

2. Risolvendo le (2) rispetto alle $\xi_{\rho h}\left(x^{\prime}\right)$ si abbia :

$$
\xi_{\rho h}\left(x^{\prime}\right)=\sum_{k=1}^{k=r} \alpha_{\rho k}(a) \frac{\partial x_{b}^{\prime}}{\partial a_{k}} \quad\left(\begin{array}{l}
b=1,2, \ldots, n \\
p=1,2, \ldots, r
\end{array}\right),
$$

donde, se con $\lambda_{1}, \lambda_{2}, \ldots, \lambda_{r}$ s'indicano $r$ quantità indeterminate, segue:

$$
\sum_{\rho=1}^{\rho=r} \lambda_{\rho} \xi_{\rho h}\left(x^{\prime}\right)=\sum_{k=1}^{k=+} \frac{\partial x_{b}^{\prime}}{\partial a_{k}} \sum_{\rho=1}^{\rho=r} \lambda_{\rho} \alpha_{\rho k}(a) \quad(b=1,2, \ldots, n) .
$$

Essendo $t$ un'altra quantita indeterminata, riguardiamo i parametri $a$ come funzioni di $t$ e delle $\lambda$, ponendo che sia, per ogni sistema di valori scelti per le $\lambda$ :

o, ciò che è lo stesso :

$$
\frac{d a_{k}}{d t}=\sum_{p=1}^{\rho=r} \lambda_{\rho} \alpha_{\rho k}(a) \quad(k=\mathrm{I}, 2, \ldots, r),
$$

$$
a_{k}=a_{k}^{(0)}+\frac{t}{\mathrm{I} !} \sum_{p=1}^{\rho=r} \lambda_{\rho} \boldsymbol{x}_{\rho k}\left(a^{0}\right)+\cdots \quad(k=\mathrm{I}, 2, \ldots, r),
$$

ove con $a^{(0)}$ indichiamo $\mathrm{i}$ valori assunti per le $a$ come corrispondenti del valore $t=8$.

Ponendo :

si può anche scrivere :

$$
t \lambda_{\rho}=\mu_{\rho} \quad(\rho=1,2, \ldots, r),
$$

$$
a_{k}=a_{k}^{(0)}+\sum_{\rho=1}^{p=r} \mu_{\rho} \alpha_{\rho k}\left(a^{0}\right)+\cdots \quad(k=1,2, \ldots, r) .
$$

Per le ipotesi fatte sulle $\psi_{\rho k}(a)$, dalle quali segue che le $x_{\rho k}(a)$ sono funzioni trascendenti intere delle variabili $a$, e che il loto determinante, che è l'inverso di quello 
delle $\psi_{\rho k}(a)$, è sempre finito e diverso da zero, si vede bene, che l'intorno del punto $a_{k}^{\circ}(k=\mathrm{I}, 2, \ldots, r)$, in cui restano definiti gl'integrali delle equazioni (7) si estende a tutto lo spazio ad $r$ dimensioni. D'altra parte le (8) definiscono le quantita $\mu$ comine funzioni delle $a$ stesse, essendo ii determinante funzionale $\frac{\partial\left(a_{1}, a_{2}, \ldots, a_{r}\right)}{\partial\left(\mu_{1}, \mu_{2}, \ldots, \mu_{r}\right)}$ non identicamente nullo, come si prova, osservando che diverso da zero è per valori nulli delle $\mu$, perchè si ha:

$$
\left(\frac{\partial a_{k}}{\partial \mu_{\rho}}\right)_{\mu=0}=\alpha_{\rho k}\left(a^{\circ}\right) \quad(k, p=\mathrm{I}, 2, \ldots, r) ;
$$

per modo che la corrispondenza che le (8) stabiliscono fra i parametri $a$ ed $i$ nuovi parametri $\mu$ è biunivoca; ed al variare comunque di questi si otterranno le trasformazioni del sistema (I) nella loro totalità.

Ciò posto dalle (6) e dalle (7) deduciamo:

Le equazioni :

$$
\sum_{\rho=1}^{\rho=r} \lambda_{\rho} \xi_{\rho b}\left(x^{\prime}\right)=\sum_{k=1}^{k=r} \frac{\partial x_{b}^{\prime}}{\partial a_{k}} \frac{d a_{k}}{d t}=\frac{d x_{b}^{\prime}}{d t} .
$$

$$
\frac{d x_{b}^{\prime}}{d t}=\sum_{\rho=1}^{\rho=r} \lambda_{\rho} \xi_{\rho b}\left(x^{\prime}\right)
$$$$
(b=1,2, \ldots, n),
$$

per ogni sistema di valori assegnati alle $\lambda$, definiscono un gruppo ad un parametro, che è il gruppo canonico, avente come trasformazione infinitesimale generatrice la:

ove si è posto:

$$
X f=\sum_{h=1}^{h=n} \xi_{h}(x) \frac{\partial f}{\partial x_{h}},
$$

$$
\xi_{h}(x)=\sum_{p=1}^{\mu=r} \lambda_{p} \xi_{p h}(x)
$$$$
(b=\mathrm{I}, 2, \ldots, n)
$$

e si vede bene che tale trasformazione infinitesimale non è altro che una combinazione lineare, con coefficienti uguali ai valori fissati per le $\lambda$, delle $r$ trasformazioni infinite simali indipendenti (4), scritte nelle variabili $x_{i}$, cioé:

Si ha infatti per le (II):

$$
X f=\sum_{\rho=1}^{\rho=r} \lambda_{\rho} X_{\rho} f
$$

$$
X f=\sum_{h=\mathrm{I}}^{h=n} \frac{\partial f}{\partial x_{h}} \sum_{\rho=1}^{\rho=r} \lambda_{\rho} \xi_{\rho h}(x)=\sum_{\rho=1}^{\rho=r} \lambda_{\rho} \sum_{h=\mathrm{I}}^{h=n} \xi_{\rho b}(x) \frac{\partial f}{\partial x_{h}},
$$

che, a causa delle (4), dimostra l'asserto.

Supponiamo che, integrando il sistema (9), si abbia :

$$
\Omega_{i}\left(x^{\prime}, \mu\right)=C_{i} \quad(i=1,2, \ldots, n) .
$$

Poichè abbiamo detto che a valori nulli dei parametri $\mu$, cioè a $t=0$, corrispondono per $\mathrm{i}$ primitivi parametri i valori $a^{(0)}$, sard in particolare:

$$
\mathbf{\Omega}_{i}\left[f\left(x, a^{\circ}\right), o\right]=C_{i}
$$$$
(i=1,2, \ldots, n),
$$

e confrontando le (I2) colle (13) si avra in ultimo:

$$
\mathbf{\Omega}_{i}\left(x^{\prime}, \mu\right)=\mathbf{\Omega}_{i}\left[f\left(x, a^{\circ}\right), \text { o }\right]
$$$$
(i=1,2, \ldots, n) .
$$ 

(IS)
Le

sono le equazioni del gruppo canonico ad un parametro, generato dalla trasformazione infinitesimale (ro).

Segue pertanto dalle (I4) che si ottiene l'insieme delle trasformazioni (I) moltiplicando la trasformazione:

$$
x_{i}^{(0)}=f_{i}\left(x, a^{0}\right)
$$

$$
(i=1,2, \ldots, n)
$$

per le trasformazioni di $\infty^{r-1}$ gruppi ad un parametro, che si hanno quando si considerino per le $\lambda$ tutti i possibili sistemi di valori.

D'altra parte, per il secondo teorema fondamentale della teoria dei gruppi s) l'insieme di questi $\infty^{t-1}$ gruppi canonici ad un parametro costituisce, a causa delle (6), un gruppo.

Si può dunque concludere che l'insieme delle trasformazioni (I) si ottiene moltiplicando la ( 16 ), che è una trasformazione qualunque di esso insieme, per le trasformazioni del gruppo $G_{r}$, generato dalle $r$ trasformazioni infinitesimali (4).

3. Ammettiamo ora che l'insieme delle trasformazioni (I) costituisca un gruppo, e consideriamo due trasformazioni qualsivogliano di esso.

Indicando queste con $S_{a}, S_{a^{\prime}}$ e con $S_{a^{(0)}}$ la (I6) sarà:

$$
S_{a}=S_{a^{(0)}} E_{\lambda}, \quad S_{a^{\prime}}=S_{a^{(0)}} E_{\mu} \text {, }
$$

ove $E_{\lambda}, E_{\mu}$ rappresentano due determinate trasformazioni di $G_{r}$; e quindi:

$$
S_{a} S_{a^{\prime}}=S_{a^{(0)}} E_{\lambda} S_{a^{(0)}} E_{\mu} \text {. }
$$

$\mathrm{Ma}$, essendo la $S_{a} S_{a^{\prime}}$ una delle trasformazioni (1), esisterà in $G_{r}$ una trasformazione $E_{y}$, per la quale si ha:

$$
S_{a} S_{a^{\prime}}=S_{a^{(0)}} E_{v} .
$$

Dal confronto di questa colla ( 17 ) si ricava che deve essere:

ossia :

$$
E_{\lambda} S_{a^{(0)}} E_{\mu}=E_{\nu}
$$

$$
S_{a^{(0)}}=E_{\lambda}^{-1} E_{\nu} E_{\mu}^{-1} \text {. }
$$

Questo ci dice che la $S_{a(0)}$, e quindi ognuna delle (1), appartiene ai gruppo $G_{r}$. E si verifica anche la proprietà inversa; perchè, indicando con $E_{\pi}$ una qualsivoglia trasformazione di $G_{r}$, si potrà sempre soddisfare all'equazione:

prendendo:

$$
S_{a^{(0)}} E_{\sigma}=E_{\pi} \text {, }
$$

$$
E_{\sigma}=S_{\mathfrak{a}^{(0)}}^{-1} E_{\pi} \text {, }
$$

che pure appartiene a $G_{r}$, il quale, essendo a coppie di trasformazioni inverse, conterrd, insieme con $S_{a^{(0)}}$, anche $S_{a^{(0)}}^{-\mathrm{z}}$.

Il gruppo rappresentato dalle (I) coincide pertanto col gruppo $G_{r}$, e, come tale, contiene la trasformazione identica.

$\mathrm{Da}$ tutto quanto è stato sin qui detto possiamo dunque, concludendo, raccogliere il seguente teorema:

5) Cfr. LIE, l. c. 
Se si ha un insieme $\infty^{r}$ di trasformazioni

$$
x_{i}^{\prime}=f_{i}(x, a) \quad(i=1,2, \ldots, n),
$$

soddisfacenti ad equazioni della forma:

$$
\frac{\partial x_{h}^{\prime}}{\partial a_{k}}=\sum_{p=1}^{\rho=r} \xi_{\rho h}\left(x^{\prime}\right) \psi_{\rho k}(a) \quad\left(\begin{array}{l}
b=1,2, \ldots, n \\
k=1,2, \ldots, r
\end{array}\right),
$$

il campo di variabilità delle $x$ e delle a essendo il campo totale di tutti $i$ possibili valori di siffatte variabili ed il determinante delle $\psi_{\rho k}($ a) risultando sempre diverso da zero, condizione necessaria e sufficiente affinchè tale insieme costituisca un gruppo è che ad esso appartenga la trasformazione identica.

4. Supponiamo ora, nelle condizioni dette sul campo di variabilita delle $x$ e delle $a$ e sul determinante delle $\psi_{o k}(a)$, di sapere soltanto che le (I) soddisfano alle equazioni (2), e ricerchiamo le condizioni, a cui in principio abbiamo accennato, equivalenti all'ipotesi che esista nell'insieme (I) la trasformazione identica, condizioni necessarie e sufficienti perchè tale insieme costituisca un gruppo.

Ammettiamo anzitutto che la träsformazione:

$$
x_{i}^{\prime \prime}=f_{i}[f(x, a), b] \quad(i=\mathrm{I}, 2, \ldots, n),
$$

prodotto di due trasformazioni qualunque:

$$
\begin{aligned}
& x_{i}^{\prime}=f_{i}(x, a) \\
& x_{i}^{\prime \prime}=f_{i}\left(x^{\prime}, b\right)
\end{aligned}
$$$$
(i=\mathrm{r}, 2, \ldots, n)
$$

delle (I), abbia soltanto $r$ parametri essenziali, il che è evidentemente necessario, se si vuole che queste costituiscano un gruppo.

Se con $c_{\rho}(\rho=1,2, \ldots, r)$ indichiamo i parametri della (I8), avremo:

$$
x_{i}^{\prime \prime}=\varphi_{i}(x, c)
$$$$
(i=1,2, \ldots, n) \text {, }
$$

e le $c$ saranno funzioni delle $a$ e delle $b$, cioè :

$$
c_{\rho}=\theta_{\rho}(a, b)
$$$$
(p=\mathrm{T}, 2, \ldots, r) \text {. }
$$

Queste relazioni risultano, come è noto, risolubili sia rispetto alle $a$, sia rispetto alle $b$. Ciò del resto segue dal fatto che, se teniamo ad esempio fisse le $a$, e facciamo variare le $b$, la (2I) percorre, come la (20), un insieme $\infty^{r}$ di trasformazioni, e percio le $r$ funzionj $c$ sono indipendenti rispetto alle $b$, ossia il determinante funzionale $\frac{\partial\left(\theta_{1}, \theta_{2}, \ldots, \theta_{r}\right)}{\partial\left(b_{1}, b_{2}, \ldots, b_{r}\right)}$ è diverso da zero. Analogamente si prova che è diverso da zero $\frac{\partial\left(\theta_{1}, \theta_{2}, \ldots, \theta_{r}\right)}{\partial\left(a_{1}, a_{2}, \ldots, a_{r}\right)}$.

Se ne ricava che, se con $S_{a}, S_{b}$ indichiamo le trasformazioni rappresentate dalle (19), (20), si potrà soddisfare alla relazione :

$$
S_{a} S_{b}=S_{a(0)}^{2}
$$

$o$, ciò che è lo stesso, alle equazioni :

$$
\theta_{\rho}(a, b)=\theta_{\rho}\left(a^{(0)}, a^{(0)}\right)
$$$$
(p=I, 2, \ldots, r)
$$

sia assegnando le $a$ e calcolando le $b$, sia assegnando le $b$ e calcolando le $a$. 
(23)

Dalla (22) abbiamo :

$$
S_{a(0)}^{-1} S_{a}=S_{a(0)} S_{b}^{-1}
$$

Dopo ciò se, come sopra, indichiamo con $E_{\lambda}$ una trasformazione generica del gruppo $G_{r}$, per il risultato a cui siamo pervenuti nel $\$$ precedente, si potrì scrivere:

$$
S_{a(0)}^{-1} S_{a}=E_{\lambda}
$$

il che ci dice che la trasformazione $S_{a^{(0)}}^{-1} S_{a}$ percorre al variare di a tutte le trasformazioni di $G_{r}$. Il medesimo si verifica allora, a causa della (23), per la trasformazione $S_{a(0)} S_{b}^{-1}$, al variare di $b$, e dell'inversa di questa $S_{b} S_{a(0)}^{-I}$, per modo che si ha:

$$
S_{b} S_{a(0)}^{-\mathfrak{x}}=E_{\mu},
$$

$E_{\mu}$ indicando ancora una trasformazione qualunque di $G_{r}$, donde :

$$
S_{b}=E_{\mu} S_{a(0)}
$$

I due insiemi di trasformazioni :

$$
S_{a(0)} E_{\lambda}, \quad E_{\mu} S_{a(0)}
$$

coincidono dunque coll'insieme dato (I), e pero possiamo simbolicamente scrivere:

che è quanto dire:

$$
S_{a(0)} G_{r}=G_{r} S_{a(0)}
$$

$$
S_{a, 0)}^{-1} G_{r} S_{a(0)}=G_{r}
$$

cioè la $S_{a(0)}$, che è una trasformazione qualunque delle (I), trasforma, sotto l'ipotesi posta al principio di questo $\$$, in sè stesso il gruppo $G_{r}$.

5. Ciò posto supponiamo ancora che all'insieme (I) appartenga il prodotto $S_{a^{(1)}} S_{a^{(2)}}$ di due sue determinate trasformazioni $S_{a(1)}, S_{a(2)}$, condizione anche questa necessaria, perchè si possa avere un gruppo.

Non escludiamo che possano $S_{a^{(x)}}, S_{a^{(2)}}$ coincidere, e, nel caso che siano distinte, nessuna ipotesi ci occorre fare sul prodotto $S_{a^{(2)}} . S_{a^{(1)}}$.

Poniamo:

$$
S_{a(1)} \cdot S_{a^{(2)}}=S_{a(3)} .
$$

Per quanto è stato detto nel $\ 2$ l'insieme delle trasformazioni ( $\mathrm{I}$ ) si potrà ottere moltiplicando una qualunque delle $S_{a^{(1)}}, S_{a^{(2)}}, S_{a_{(3)}}$ per le trasformazioni di $G_{r}$.

Essendo allora $S_{a}, S_{a}$ due trasformazioni qualsivogliano di detto insieme, sarà :

$$
S_{a}=S_{a^{(1)}} E_{\lambda}^{(1)}, \quad S_{a^{\prime}}=S_{a^{(2)}} E_{\mu}^{(2)}
$$

ove $E_{\lambda}^{(1)}, E_{\mu}^{(2)}$ rappresentano due determinate trasformazioni di $G_{r}$, i cui parametri $\mu$ sono dati, per la prima dal sistema di equazioni:

$$
a_{k}=a_{k}^{(\mathrm{r})}+\frac{\mathrm{I}}{\mathrm{I} !} \sum_{\rho=1}^{\rho=r} \mu_{\rho} x_{p k}\left(a^{(\mathrm{s})}\right)+\cdots \quad(k=1,2, \ldots, r),
$$

e per l'altra dal sistema di equazioni:

$$
a_{k}^{\prime}=a_{k}^{(2)}+\frac{\mathrm{I}}{\mathrm{I} !} \sum_{\rho=1}^{p=r} \mu_{\rho} \alpha_{\rho k}\left(a^{(2)}\right)+\cdots \quad(k=\mathrm{I}, 2, \ldots, r) .
$$

Si consideri della $E_{\lambda}^{(1\}}$ la trasformata per mezzo della $S_{a^{(2)}}$, che pure appartiene a $G_{r}$ 
( $\left(\right.$ prec.), e s'indichi con $\bar{E}_{\lambda}^{(j)}$, si ponga cioè :

$$
\bar{E}_{\lambda}^{(\mathrm{r})}=S_{a^{(2)}}^{-\mathrm{I}} E_{\lambda}^{(\mathrm{r})} S_{a^{(2)}}
$$

La $S_{a^{(3)}} \bar{E}_{\lambda}^{(\mathrm{t})} E_{\mu}$ apparterrà $\left(\S_{2}\right)$ all'insieme (I): i suoi parametri si otterranno dalle formole :

$$
a_{k}=a_{k}^{(3)}+\sum_{\rho=1}^{\rho=r} \mu_{\rho} \alpha_{p k}\left(a^{(3)}\right) \ldots \quad(k=1,2, \ldots, r),
$$

sostituendo nel secondo membro al posto delle $\mu$ i valori corrispondenti alla trasformazione $\bar{E}_{\lambda}^{(1)} E_{\mu}$.

La medesima trasformazione $S_{n}(\xi) \bar{E}_{\lambda}^{(1)} E_{\mu}$ rappresenta inoltre il prodotto delle date trasformazioni $S_{a}, S_{a^{\prime}}$, perchè si ha dalle (25):

donde per la (26):

$$
S_{a} \cdot S_{a^{\prime}}=S_{a^{(1)}} E_{\lambda}^{(1)} S_{a^{(2)}} E_{\mu}^{(2)},
$$

e finalmente:

$$
S_{a} \cdot S_{a^{\prime}}=S_{a^{(1)}} S_{a^{(2)}} \bar{E}_{\lambda}^{(1)} E_{\mu}^{(2)}
$$

$$
S_{a} \cdot S_{a^{\prime}}=S_{a^{(3)}} \bar{E}_{\lambda}^{(1)} E_{\mu}^{(2)} \text {. }
$$

É ovvio che in modo analogo si perviene a dimostrare che all'insieme (I) appartiene anche il prodotto $S_{a^{\prime}} . S_{a}$ delle due assegnate trasformazioni: basta perciò esprimere $S_{a^{\prime}}$ ed $S_{a}$ nel modo dianzi tenuto rispettivamente per $S_{a}$ ed $S_{a^{\prime}}$.

Le considerazioni svolte in questo e nel precedente $\$ permettono di enunciare il seguente teorema:

Se si ba un insieme $\infty^{r}$ di trasformazioni:

$$
x_{i}^{\prime}=f_{i}(x, a)
$$

$$
(i=1,2, \ldots, n) \text {, }
$$

soddisfacenti ad equazioni della forma:

$$
\frac{\partial x_{b}^{\prime}}{\partial a_{k}}=\sum_{\rho=1}^{\rho=r} \xi_{\rho h}\left(x^{\prime}\right) \Psi_{\rho k}(a) \quad\left(\begin{array}{l}
b=1,2, \ldots, n \\
k=1,2, \ldots, r
\end{array}\right),
$$

il campo di variabilità delle $x$ e delle a essendo il campo totale di tutti i possibili valori di siffatte variabili, ed il determinante delle $\psi_{\rho k}(a)$ risultando sempre diverso da zero, condizione necessaria e sufficiente affinchè tale insieme costituisca un gruppo è che la trasformazione prodotto di due qualsivogliano di esse abbia soltanto $r$ parametri essenziali,e per un particolare sistema di valori di questi coincida con una delle (27).

6. La condizione espressa dalla (24), necessaria, come si è visto, perchè i parametri essenziali, da cui dipende la trasformazione prodotto di due qualsivogliano delle (I), siano soltanto $r$, è anche sufficiente, perchè da essa si ricava che coll'insieme delle trasformazioni (I) coincide ognuno degli insiemi:

$$
S_{a(0)} E_{\lambda}, \quad E_{\mu} S_{a^{(0)}},
$$

e che essendo pertanto il prodotto di due qualsivogliano delle (I) espresso da

$$
S_{a(0)} E_{\lambda} E_{\mu} S_{a^{(0)}},
$$

esso contiene $i$ soli $r$ parametri essenziali di $E_{\lambda} E_{\mu}$.

Si può quindi anche dire: 
Nelle ipotesi del teorema precedente, condizione necessaria e sufficiente affinchè l'insieme dato di trasformazioni costituisca un gruppo è che ad esso appartenga il prodotto $S_{a^{(1)}} S_{a^{(2)}}$ di due sue determinate trasformazioni $S_{a^{(1)}}, S_{a^{(2)}}$ ed una trasformazione $S_{a}$ (che potrebbe anche coincidere con una delle precedenti) per la quale resti trasformato in sè il gruppo $G_{r}$, generato dalle $r$ trasformazioni infinitesimali:

$$
X_{\rho} f=\sum_{h=1}^{h=n} \xi_{\rho h}(x) \frac{\partial f}{\partial x_{b}} \quad(p=1,2, \ldots, r) .
$$

7. La condizione, cui deve soddisfare la $S_{a}^{-}$, è soddisfatta, se il sistema di queste trasformazioni infinitesimali è, rispetto ad essa, invariante, se cioè posto:

si ha:

$$
\bar{x}_{i}=f_{i}(x, \bar{a})
$$$$
(i=1,2, \ldots, n)
$$

$$
\sum_{\rho=1}^{\rho=r} \varepsilon_{\rho} \sum_{b=1}^{h=n} \xi_{\rho h}(x) \frac{\partial f}{\partial x_{h}}=\sum_{p=1}^{\rho=r} e_{\rho}^{-h=n} \sum_{h=1}^{h=1} \xi_{\rho h}(\bar{x}) \frac{\partial f}{\partial \bar{x}_{h}},
$$

ove le $\bar{e}_{\rho}$ sono funzioni delle sole $\varepsilon_{\rho}$, perchè allora la $S_{\bar{a}}$ trasforma, come è noto, l'uno nell'altro gli $\infty^{r-1}$ gruppi ad un parametro, che costituiscono $G_{r}$. D'altra parte, se le (I) costituiscono un gruppo, coincidendo questo con $G_{r}$, rispetto ad ognuna delle (I) è invariante il sistema delle (28).

Cio permette di concludere che l'insieme dato delle trasformazioni (I) costituisce un gruppo, allora e solo allora quando ad esso appartiene il prodotto $S_{a^{(2)}} . S_{a^{(2)}}$ di due sue - determinate trasformazioni $S_{a^{(1)}}, S_{a^{(2)}}$, ed una trasformazione $S_{\bar{a}}$ (che potrebbe anche coincidere con una delle precedenti) rispetto alla quale $\dot{e}$ invariante il sistema delle $r$ trasformazioni infinitesimali (28).

Catania, marzo 1907.

C. SEverini. 